\title{
Successful control of Johne's disease in nine dairy herds: Results of a six-year field trial
}

\author{
M. T. Collins, ${ }^{1}$ V. Eggleston, and E. J. B. Manning \\ Department of Pathobiological Sciences, School of Veterinary Medicine, University of Wisconsin-Madison, Madison 53706-1102
}

\begin{abstract}
The objective was to evaluate if a standardized Johne's disease control program significantly reduced the prevalence of cattle infected with Mycobacterium avium ssp. paratuberculosis in dairy herds with a moderate to high initial infection prevalence of $\geq 10 \%$ ELISApositive adult cattle. Nine Wisconsin dairy herds of diverse sizes and management styles completed the 6-yr study. The control program involved changes to heifer rearing practices in combination with a routine testing program. For heifers, the program specifically required 1) segregated maternity pens for ELISA-positive and ELISA-negative cattle; 2) removal of calves from the maternity pen in $<2 \mathrm{~h} ; 3$ ) use of colostrum only from individual ELISA-negative cows (no colostrum pooling); 4) hygienic collection of colostrum; 5) feeding of pasteurized milk as milk replacer or on-farm pasteurized milk until weaning; and 6) minimizing contact with manure from the adult cattle until weaning. The testing program was designed to detect the most infectious cattle by using a commercial ELISA once on every adult during each lactation. Producers were required to cull cows with strong-positive ELISA results before the next calving and to label cows with low- to medium-level ELISA results and manage them to limit infection transmission. Outcomes were measured by comparing the apparent prevalence based on ELISA or fecal culture in the whole herd and in first-lactation cohorts at 2 time points: before implementation of the control program and at the end of the trial. The combined results from the 9 herds showed a significant reduction in ELISA-positive cows, from $11.6 \%$ at the start of the trial to $5.6 \%$ at conclusion of the trial. The apparent prevalence decline among first-lactation cows was greater and was evident by ELISA (10.4 vs. $3.0 \%)$ and by fecal culture (17.0 vs. 9.5\%). Although variations among farms were observed, the collective results demonstrated that bovine paratuberculosis can be controlled in dairy herds through effective heifer husbandry practices in combination with diagnostic
\end{abstract}

Received August 21, 2009.

Accepted December 18, 2009.

${ }^{1}$ Corresponding author: mcollin5@wisc.edu testing to identify, for culling or management, cows most likely infectious.

Key words: Johne's disease, paratuberculosis, control, ELISA

\section{INTRODUCTION}

Johne's disease, also known as paratuberculosis, is a chronic, granulomatous, gastrointestinal tract disease of cattle and other ruminants caused by Mycobacterium avium ssp. paratuberculosis (M. paratuberculosis). The infection has been documented worldwide (Harris and Barletta, 2001; Manning, 2001; Nielsen and Toft, 2009), and a 2007 USDA survey indicated that infected animals are present in at least $68 \%$ of US dairy herds (USDA-APHIS-VS-CEAH, 2008).

The incubation period is typically prolonged, from the initial calfhood infection followed by numerous clinically normal years to its end in disease (diarrhea, rapid weight loss, low milk production) and death when cows are $5 \mathrm{yr}$ or older (Manning and Collins, 2001; Tiwari et al., 2006). During the clinically normal incubation stage, infected cows shed the causative organism in their manure and milk, thus transmitting the infection to multiple generations of their own and herdmates' calves and contaminating the environment.

Recommended methods for control of Johne's disease involve a two-pronged approach: 1) employ animal husbandry practices to limit opportunities for M. paratuberculosis transmission to calves, and 2) simultaneously test adults to identify the most infectious, heavy fecal shedders of $M$. paratuberculosis in the herd (Nielsen, 2009). Infectious cattle can be culled from the herd or managed to limit their direct or indirect contact with calves (i.e., discard colostrum and calve in pens separate from the test-negative herd members). These recommendations are well accepted, and selected components of such a control program have been evaluated epidemiologically (Stabel, 2008; Tiwari et al., 2009; Whittington and Windsor, 2009). Yet, no longitudinal studies have measured the net effect of a specific control program on the within-herd prevalence of $M$. paratuberculosis infections in commercial dairy herds. This study hypothesizes that implementing Johne's disease control 
Table 1. Characteristics of the 9 study herds at the start of the trial in 2002

\begin{tabular}{|c|c|c|c|c|c|c|c|c|c|}
\hline Item & \multicolumn{9}{|c|}{ Herd } \\
\hline Breed & Jersey & Holstein & Holstein & Holstein & Jersey & Holstein & Holstein & Holstein & Holstein \\
\hline Housing system & Freestall & Freestall & Stanchion & Freestall & Stanchion & Freestall & Freestall & Freestall & Stanchion \\
\hline Risk assessment score ${ }^{1}$ & 26 & 35 & 43 & 48 & 34 & 34 & 39 & 21 & 40 \\
\hline ELISA-positive, ${ }^{2} \%$ & 17.6 & 13.4 & 11.2 & 13.4 & 12.6 & 16.3 & 9.8 & 10.1 & 20.9 \\
\hline
\end{tabular}

${ }^{1}$ Risk assessment scores were based on the standardized system used in the US in 2002 (USAHA Risk Assessment, 2003). All herds were scored by the same person (VE). These scores are low although no guidelines have been established for score interpretation.

${ }^{2}$ ELISA for serum antibody to paratuberculosis applied to all cows in the herd.

calf rearing practices in conjunction with regular use of a low-cost blood test to identify and manage the most infectious cows affordably decreases within-herd prevalence of Johne's disease.

Nine dairy herds of diverse sizes and management styles followed the same control program for $6 \mathrm{yr}$. Compliance was monitored by regular visits to every herd multiple times each year. The control program's effect was judged based on a comparison of within-herd apparent (test) prevalence of age-matched cohorts before and after full implementation of the control program. Effective control was defined as a significant decrease in within-herd paratuberculosis prevalence; eradication of the infection was not a goal.

\section{MATERIALS AND METHODS}

\section{Study Herds}

Dairy herds were recruited in Wisconsin by veterinary practitioners in the state. Practitioners were asked to nominate herds with significant Johne's disease problems with the permission of the herd owner. There were 2 criteria to qualify: 1) $\geq 10 \%$ ELISA-positive (blood samples) cows, and 2) the herd owner's willingness to sign a contract agreeing to implement the prescribed Johne's disease control program for the duration of the project. Thirty herds were nominated and evaluated. Ten herds were ultimately selected and the herd owners signed contracts. Nine herds complied with the program rules and completed the 6-yr study (Table 1 ).

\section{Johne's Disease Control Program}

Herd Management. Herd management changes focused on affordable and easy-to-implement heifer calf rearing methods designed to limit chances for $M$. paratuberculosis transmission. The following 7 specific changes were required of herd owners:

1. Maternity pens must be segregated. Only ELISAnegative cows were allowed to calve in the "clean" pen and a high standard of hygiene should be maintained in this pen. Neither ELISA-positive or sick cows were allowed in this pen.

2. Calves must be promptly (ideally within $2 \mathrm{~h}$ of birth) removed from the maternity pen or in some other way effectively isolated from exposure to cow manure.

3. Calves must be fed by bottle or esophageal tube $4 \mathrm{~L}$ (3 L for Jersey calves) of colostrum from a single ELISA-negative cow (colostrum pooling not allowed) within $6 \mathrm{~h}$ of birth. Colostrum should be hygienically collected to limit manure contamination. Hygienic collection entailed teat preparation as per industry standards before milking (washing, rinsing, and drying, antiseptic dip) and collection into a clean vessel for feeding.

4. No unpasteurized milk can be fed to the calves. Until weaning, calves must receive either commercial milk replacer or on-farm pasteurized milk.

5. Calves must be housed away from the adult herd and managed to limit, if not completely prevent, exposure to manure from cows.

6. Cows with ELISA results classified as "strongpositive" must be culled from the herd when economically optimal, but always before the next calving. Cows with low- to medium-level ELISA results must be visibly labeled (most often by addition of a plastic strip to an existing ear tag, but some herds used colored leg bands).

7. ELISA-negative cows calving in the "clean" pen are used as colostrum donors and their heifer calves favored for selection as herd replacements.

Herd owners were asked not to purchase herd replacements for the duration of the project; however, the design goal was compromised for practical reasons in several herds, and herd replacements were allowed after consultation with the project investigators. The 
purchased cattle were not included in the herd paratuberculosis test data analysis.

Herd Testing. Management of a cow, her milk and colostrum, and calf was affected by her Johne's disease diagnostic test status. The paratuberculosis testing program was based on measurement of serum antibodies to $M$. paratuberculosis using a commercial ELISA kit (IDEXX Laboratories, Inc., Westbrook, ME). Every cow was tested once during each lactation. The time during lactation cycles when cows were tested was at the option of the herd owner; some herd owners elected to test cows before breeding (cows with strong-positive ELISA results labeled "do not breed"), whereas others favored testing cows at the end of their lactation. The ELISA results were reported to herd owners, and cows were ranked in descending order by the magnitude of the test result, sample/positive (optical density (OD) of sample - OD negative control)/(OD positive control - OD negative control) value (Collins, 2002; Collins et al., 2005).

Fecal samples were collected at the time of blood sample collection. Fecal samples were processed for isolation of $M$. paratuberculosis by using culture-based methods (Collins et al., 1990). Fecal culture results were not reported to the herd owners but used only as an independent measure of within-herd infection rate.

Program Compliance Monitoring. The project manager (VE) visited every herd at least 3 or 4 times/ yr. At the first visit, a herd risk assessment was performed (USAHA Risk Assessment, 2003). This standardized risk assessment system adopted in the United States systematically evaluated 6 management areas of dairy operations for likelihood of M. paratuberculosis transmission, resulting in a summary score and ranking of those areas needing modification to limit transmission risks. The prescribed Johne's disease control program was designed to address management areas most commonly needing improvement on dairy farms. At subsequent visits, herds were evaluated for compliance with the Johne's disease control program specified in the contract. Herd management practices were evaluated by visual inspection during a walk-through of the dairy and discussions with herd owners and their employees. Herd DHI records were examined to document that cows with strong-positive ELISA results were being culled.

\section{Data Analysis}

Changes in within-herd apparent prevalence before and after full implementation of the Johne's disease control program were based on ELISA and fecal culture and statistically evaluated using the 2 -tailed $t$-test, with $P$-values $<0.05$ considered significant (Remington and Schork, 1970). Animal cohorts compared were the entire adult herd (all cows, lactating or dry, that had calved at least once) and first-parity (lactation) cows. Only those heifers born after full implementation of the control program were used as a cohort for comparison to those born and raised on the farm before implementation of the program (i.e., purchased replacement cattle were excluded from the analysis). Results were evaluated for each herd individually and for all 9 herds combined.

\section{RESULTS}

\section{Johne's Disease Control Program Implementation and Compliance}

The dairy herds were diverse in size, location in Wisconsin, management system, and labor and financial resources available to implement the control program. The apparent (ELISA) prevalence for the adult herd at the start of the project ranged from 10.1 to $20.9 \%$ (Table 1). Herd owners were given latitude in precisely how the required animal husbandry changes were implemented on their farm. Ultimately, the study manager (VE) judged whether herd owners complied with the intent of the control program. It took roughly 6 mo from signing the contract for study participation to full implementation of the control program.

\section{Apparent Prevalence at the Herd Level}

Eight of the 9 herds had a significant decrease in within-herd $M$. paratuberculosis infection rate (apparent prevalence) based on the percentage of the adult herd that was ELISA-positive (Figure 1). The combined results from all 9 herds showed that 410/3,537 (11.6\%) of cows were ELISA-positive at the start of the trial, whereas $188 / 3,344(5.6 \%)$ were ELISA-positive at conclusion of the trial $(P<0.0001)$. Whole-herd apparent prevalence by fecal culture significantly decreased in 2 of the 9 herds (herd $\mathrm{B},-3.8 \%$ and herd $\mathrm{H},-2.6 \%$; $P$ $<0.05$ ), but this decrease was not seen when data from all 9 herds were combined.

\section{Apparent Prevalence by Lactation Cohort}

First-lactation cows were the first age cohort available for comparison in which all individual animals were born either before or after full implementation of the control program. By ELISA, the apparent prevalence of paratuberculosis declined significantly in 4 of the 9 herds (B, F, G, and H; $P<0.05)$ and for all herds combined $(P<0.0001$; Figure 2$)$. Fecal culture apparent prevalence significantly declined for the first-lactation 


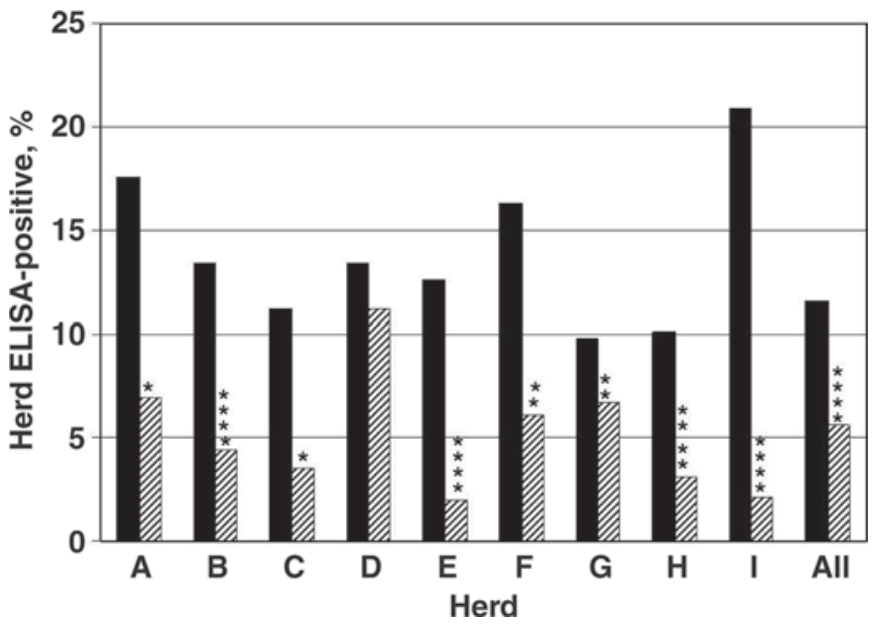

Figure 1. Percentage of cows testing ELISA-positive for each herd individually and all herds combined before (solid bars) and at the end (striped bars) of the control program $\left({ }^{*} P<0.05 ;{ }^{* *} P<0.01\right.$; ${ }^{* * * *} P$ $<0.0001)$.

cohort in 3 of the 9 herds $(\mathrm{B}, \mathrm{F}$, and $\mathrm{G} ; P<0.05)$ and for all herds combined $(P<0.01$; Figure 3$)$. Insufficient numbers of cows had finished their second lactation before conclusion of the study to make valid statistical comparisons of the apparent prevalence in this cohort.

\section{DISCUSSION}

The costs of intervention to control Johne's disease must be balanced against the costs of the disease (Pillars et al., 2009). Johne's disease reduces milk production, herd life, fertility, and carcass weight to some degree at any prevalence level, but the disease is not perceived by producers as worth addressing until the infection rate

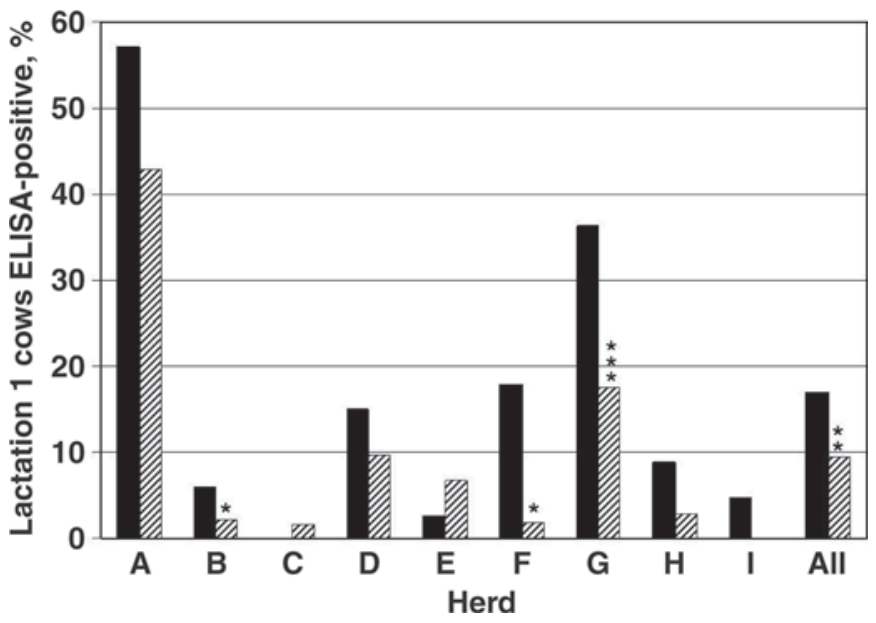

Figure 2. Percentage of cattle in their first lactation testing ELISApositive for each herd individually and all herds combined before (solid bars) and at the end (striped bars) of the control program $\left({ }^{*} P<0.05\right.$; ** $P<0.01$; *** $P<0.001)$. in a herd becomes high and frequent cases of clinical Johne's disease are seen as a cause of excessive culling.

A comparison of the paratuberculosis apparent prevalence in dairy cattle before and after implementation of the specific Johne's disease control program among all 9 study herds showed a significant decline in $M$. paratuberculosis infection rate based on ELISA for serum antibodies in all cows (11.6 vs. $5.6 \% ; P<0.0001)$, ELISA in the first-lactation cohort (10.4 vs. $3.0 \% ; P<0.0001$ ), and fecal culture on the first-lactation cohort (17.0 vs. $9.5 \% ; P<0.01)$. Because an insufficient number of animals born after implementation of the control program completed their second lactation before termination of the trial, this age cohort could not be analyzed. Epidemiological and economic models of paratuberculosis suggest that the benefits of lower within-herd prevalence could be amplified the longer the control program continued because of continual lowering of the infection pressure in the herd (Collins and Morgan, 1992; Groenendaal et al., 2002; Groenendaal and Galligan, 2003). Funding limitations to sustain the study beyond $6 \mathrm{yr}$ made testing this assumption impossible.

Eight of the 9 study herds had a significant decrease in the percentage of the herd (all cows) that was either ELISA-positive or fecal culture-positive, based on whole-herd tests before the control program compared with test results in the last year of the program $(P<$ $0.05)$. Variations in the magnitude of decline in apparent prevalence among herds could have resulted from numerous factors including stage of the paratuberculosis epidemic, Johne's disease control efforts before start of the project (herd $\mathrm{C}$ had culled some test-positive cows just before start of the trial), level of contamination on

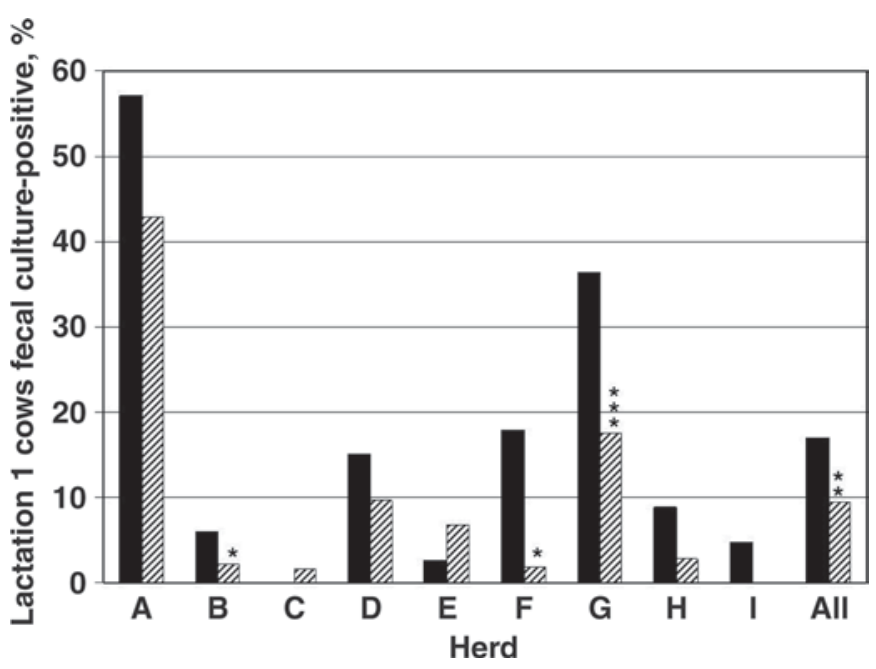

Figure 3. Percentage of cattle in their first lactation testing fecal culture-positive for each herd individually and all herds combined before (solid bars) and at the end (striped bars) of the control program $\left({ }^{*} P<0.05 ;{ }^{* *} P<0.01 ;{ }^{* * *} P<0.001\right)$ 
the premises, virulence of the M. paratuberculosis strain infecting the particular herd, or differences in genetic susceptibility of the cows (Gonda et al., 2006).

Several subjective observations about the Johne's control program are worth noting. There was no apparent difference in Johne's disease control success between herds that fed milk replacer and those that used on-farm pasteurizers. Second, improvement in colostrum collection and storage procedures was needed for most herds. In particular, colostrum was often not being collected hygienically. Last, maternity pen management was the most challenging area for dairy operations to change. Individual calving pens were not feasible for most herds, but designation of one pen for ELISA-negative cows and another for ELISA-positive cows was achievable. Removal of calves from the maternity pen or at least to an area inside the pen that was free of adult manure contamination (e.g., a "safe zone") within $2 \mathrm{~h}$ of birth was easier to accomplish in large herds than in smaller herds as it generally required additional labor for closer pen monitoring. This cost was more affordable for the large herds.

Transmission risk-based culling relied on the proven strong association between the magnitude of ELISA result and the probability a cow is shedding $M$. paratuberculosis (Collins, 2002; Collins et al., 2005). The control program tested in this study restricted expensive corrective actions, such as culling, to the cows that were most infectious (Collins, 2002), least likely to survive another lactation (Lombard et al., 2005), and probably carrying a fetus that had been infected as a result of infection dissemination in the cow (Sweeney et al., 1992; Antognoli et al., 2008). A crucial facet of the control program was the option for owners to retain cows with low- or medium-level ELISA results to generate milk income, but that could be managed to avoid infection transmission to calves. This aspect of the control program required diligence on the part of herd managers because these cows were almost always fecal shedders of $M$. paratuberculosis. The overall success of the control program indicated that herd owners and their employees were able to successfully manage these ELISA-positive cows. Knowledge that cows were low- or medium-level ELISA-positive for paratuberculosis may have resulted in earlier culling from the herd, particularly if these cows developed other health problems or if milk production declined (anecdotal observations from producers).

Johne's disease has been shown to affect milk production when evaluated at the cow level (Lombard et al., 2005; Nielsen et al., 2009; Smith et al., 2009). When milk production was evaluated based on monthly DHI data using multiple traits over the 6-yr trial, no consistent association between apparent prevalence of paratuberculosis and milk production at the herd level was found (data not shown). Milk production at the herd level was affected by so many diverse factors that the specific effect of higher production resulting from fewer cows infected with $M$. paratuberculosis could not be detected.

A goal achieved was to determine if Johne's disease could be controlled using low-cost intervention strategies in a diverse group of commercial dairy herds. Specifically, simple changes to heifer rearing practices in line with industry standards for best management practices, a low-cost diagnostic test for paratuberculosis (ELISA), cow culling limited to only those cows considered most infectious (i.e., testing strong-positive by ELISA), and retention in the herd but careful management of other ELISA-positive cows were efficacious. The control program was effective in reducing withinherd apparent prevalence while limiting costs of managing the infection, essentially validating the risk-based control program being using in Denmark (Kudahl et al., 2008; Nielsen, 2009). The study corroborates observations made in Michigan and Minnesota on Johne's disease control programs in general (Wells et al., 2008; Pillars et al., 2009).

Faster paratuberculosis control could be achieved with greater effort and investment in management changes, use of a more sensitive diagnostic test, and more aggressive culling of test-positive animals and their daughters. Such efforts and greater investments were difficult for many producers to sustain and this study promoted a program that was financially manageable for most producers and produced measurable benefits. The program design was based on a published cost-benefit analysis of paratuberculosis control for commercial dairy herds that showed that low-cost tests were favored over high-cost and higher sensitivity tests (Dorshorst et al., 2006). Paratuberculosis control, not paratuberculosis eradication, was the objective.

Because an association between the test status of a dam and her offspring was observed for only 1 of the 9 herds (data not shown), it is hard to support a recommendation to cull all daughters of test-positive cows. Of course, by culling cows with strong-positive ELISA results before they calve again on the farm, daughters are effectively culled. Daughters of cows with advanced M. paratuberculosis infections more likely acquired the infection in utero (Whittington and Windsor, 2009).

A greater number of infectious cows would have been detected by use of fecal culture instead of ELISA (Collins et al., 2005). Economic decision analysis indicates that for commercial dairy herds, use of a more sensitive but more expensive test is not cost-effective for a control program (Dorshorst et al., 2006). Seven of the 9 herds provided samples for a study evaluating ELISA 
accuracy (Collins et al., 2005), including the assay used to support the control program. The reference test in that study was fecal culture done in parallel by 3 independent laboratories. They demonstrated that of 118 cows classified as moderate to heavy shedders (fecal shedding level of 2.1 to 4.0$), 86(72.9 \%)$ were detected by the ELISA; that is, there were 32 false-negative ELISA results. For 297 cows classified as low shedders (fecal shedding level of 0.1 to 2.0 ), 41 (13.8\%) were ELISA-positive; that is, there were 256 false-negative ELISA results. This study highlights the fact that control of paratuberculosis was successfully achieved in spite of the high rate of false-negative ELISA results. Limitations of the study were those inherent in any field trial; that is, lack of complete control over how herds were managed on a daily basis and the degree of variability in diagnostic test results among the 9 herds. Nevertheless, a significant decline in M. paratuberculosis infection prevalence was observed overall in spite of the study limitations, attesting to the importance of the findings and ability to extrapolate the results to the general population of dairy herds in the United States.

\section{ACKNOWLEDGMENTS}

This project was supported in part by the Wisconsin Milk Marketing Board (Madison), USDA-APHIS-VS cooperative agreements, the Wisconsin Agriculture Experiment Station, the University of Wisconsin, and the Johne's Testing Center (Madison, WI). Special thanks are owed to the 9 dairy producers who allowed their herds to be used and invested both time and money to implement the Johne's disease control program.

\section{REFERENCES}

Antognoli, M. C., F. B. Garry, H. L. Hirst, J. E. Lombard, M. M. Dennis, D. H. Gould, and M. D. Salmon. 2008. Characterization of Mycobacterium avium subspecies paratuberculosis dissemination in dairy cattle and its association with antemortem test results. Vet. Microbiol. 127:300-308.

Collins, M. T. 2002. Interpretation of a commercial bovine paratuberculosis enzyme-linked immunosorbent assay by using likelihood ratios. Clin. Diagn. Lab. Immunol. 9:1367-1371.

Collins, M. T., K. B. Kenefick, D. C. Sockett, R. S. Lambrecht, J. McDonald, and J. B. Jørgensen. 1990. Enhanced radiometric detection of Mycobacterium paratuberculosis using filter concentrated fecal specimens. J. Clin. Microbiol. 28:2514-2519.

Collins, M. T., and I. R. Morgan. 1992. Simulation model of paratuberculosis control in a dairy herd. Prev. Vet. Med. 14:21-32.

Collins, M. T., S. J. Wells, K. R. Petrini, J. E. Collins, R. D. Schultz, and R. H. Whitlock. 2005. Evaluation of five antibody detection tests for bovine paratuberculosis. Clin. Diagn. Lab. Immunol. 12:685-692.

Dorshorst, N. C., M. T. Collins, and J. E. Lombard. 2006. Decision analysis model for paratuberculosis control in commercial dairy herds. Prev. Vet. Med. 75:92-122.

Gonda, M. G., Y. M. Chang, G. E. Shook, M. T. Collins, and B. W. Kirkpatrick. 2006. Genetic variation of Mycobacterium avium ssp. paratuberculosis infection in US Holsteins. J. Dairy Sci. 89:18041812.
Groenendaal, H., and D. T. Galligan. 2003. Economic consequences of control programs for paratuberculosis in midsize dairy farms in the United States. J. Am. Vet. Med. Assoc. 223:1757-1763.

Groenendaal, H., M. Nielen, A. W. Jalvingh, S. H. Horst, D. T. Galligan, and J. W. Hesselink. 2002. A simulation of Johne's disease control. Prev. Vet. Med. 54:225-245.

Harris, N. B., and R. G. Barletta. 2001. Mycobacterium avium ssp. paratuberculosis in veterinary medicine. Clin. Microbiol. Rev 14:489-512.

Kudahl, A. B., S. S. Nielsen, and S. Ostergaard. 2008. Economy, efficacy, and feasibility of a risk-based control program against paratuberculosis. J. Dairy Sci. 91:4599-4609.

Lombard, J. E., F. B. Garry, B. J. McCluskey, and B. A. Wagner. 2005. Risk of removal and effects on milk production associated with paratuberculosis status in dairy cows. J. Am. Vet. Med. Assoc. 227:1975-1981.

Manning, E. J. 2001. Mycobacterium avium subspecies paratuberculosis: A review of current knowledge. J. Zoo Wildl. Med. 32:293-304.

Manning, E. J. B., and M. T. Collins. 2001. Mycobacterium avium subsp. paratuberculosis: pathogen, pathogenesis and diagnosis. Rev. Sci. Tech. 20:133-150.

Nielsen, S. S. 2009. Use of diagnostics for risk-based control of paratuberculosis in dairy herds. In Practice 31:150-154.

Nielsen, S. S., M. A. Krogh, and C. Enevoldsen. 2009. Time to the occurrence of a decline in milk production in cows with various paratuberculosis antibody profiles. J. Dairy Sci. 92:149-155.

Nielsen, S. S., and N. Toft. 2009. A review of prevalences of paratuberculosis in farmed animals in Europe. Prev. Vet. Med. 88:1-14.

Pillars, R. B., D. L. Grooms, C. A. Wolf, and J. B. Kaneene. 2009. Economic evaluation of Johne's disease control programs implemented on six Michigan dairy farms. Prev. Vet. Med. 90:223-232.

Remington, R. D., and M. A. Schork. 1970. Hypothesis testing. Pages 192-228 in Statistics with Applications to the Biological and Health Sciences. Prentice-Hall Inc., Englewood Cliffs, NJ.

Smith, R. L., Y. T. Grohn, A. K. Pradhan, R. H. Whitlock, J. S. Van Kessel, J. M. Smith, D. R. Wolfgang, and Y. H. Schukken. 2009. A longitudinal study on the impact of Johne's disease status on milk production in individual cows. J. Dairy Sci. 92:2653-2661.

Stabel, J. R. 2008. Pasteurization of colostrum reduces the incidence of paratuberculosis in neonatal dairy calves. J. Dairy Sci. 91:36003606.

Sweeney, R. W., R. H. Whitlock, and A. E. Rosenberger. 1992. Mycobacterium paratuberculosis isolated from fetuses of infected cows not manifesting signs of the disease. Am. J. Vet. Res. $53: 477-480$.

Tiwari, A., J. A. VanLeeuwen, I. R. Dohoo, G. P. Keefe, J. P. Haddad, H. M. Scott, and T. Whiting. 2009. Risk factors associated with Mycobacterium avium subspecies paratuberculosis seropositivity in Canadian dairy cows and herds. Prev. Vet. Med. 88:32-41.

Tiwari, A., J. A. VanLeeuwen, S. L. B. McKenna, G. P. Keefe, and H. W. Barkema. 2006. Johne's disease in Canada. Part I: Clinical symptoms, pathophysiology, diagnosis, and prevalence in dairy herds. Can. Vet. J. 47:874-882.

USAHA (US Animal Health Association) Risk Assessment. 2003. Handbook for Veterinarians and Dairy Producers. A guide for Johne's disease risk assessment and management plans for dairy herds. 3rd ed. Herd Management and Education Standards Task Force for the Voluntary Bovine Johne's Control Program. USDA, Riverdale, MD.

USDA-APHIS-VS-CEAH. 2008. Pages 1-4 in Johne's disease on U.S. dairies, 1991-2007.USDA-APHIS-VS-CEAH, Ft. Collins, CO.

Wells, S. J., W. L. Hartmann, and P. L. Anderson. 2008. Evaluation of progress made by dairy and beef herds enrolled in the Minnesota Johne's Disease Control Program. J. Am. Vet. Med. Assoc. 233:1920-1926.

Whittington, R. J., and P. A. Windsor. 2009. In utero infection of cattle with Mycobacterium avium ssp. paratuberculosis: A critical review and meta-analysis. Vet. J. 179:60-69. 